\title{
Interferência do volume de aplicação e adjuvantes nos depósitos da
}

\section{pulverização em plantas de feijão}

Interference of spray volume and adjuvant on bean plants spray deposits Interferencia del volumen de aplicación y adyuvantes en los depósitos de la pulverización en plantas de frijol

Maurício Gomes Azevedo Discente do curso de Engenharia Agronômica, FCAT - UNESP, Brasil mauriciogazevedo@hotmail.com

Pedro Hideki Omoto Discente do curso de Engenharia Agronômica, FCAT - UNESP, Brasil pe_omoto19@hotmail.com

\section{Evandro Pereira Prado}

Professor Assistente Doutor, FCAT - UNESP, Brasil epprado@dracena.unesp.br 
RESUMO

Adjuvantes são substancias adicionadas na calda de pulverização cuja finalidade é melhorar a eficiência das aplicações de defensivos agrícolas. A pesquisa teve como objetivos avaliar diferentes classes de adjuvantes agrícolas e volume de aplicação sobre os depósitos da pulverização em plantas de feijão. $O$ ensaio para determinação do efeito do adjuvante na retenção da calda de pulverização foi realizado em estufa e o ensaio visando à interferência do volume de aplicação em condições de campo. Resultados distintos em relação à deposição da calda em plantas de feijão foram encontrados a depender do tipo de adjuvante utilizado. $O$ adjuvante Haiten aplicado na dose de 100

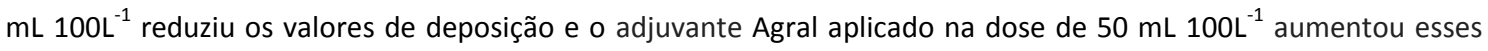
valores. O aumento do volume de aplicação proporcionou maiores valores de deposição nas plantas de feijão, porém esse aumento foi significativo até o volume de $160 \mathrm{~L} \mathrm{ha}{ }^{-1}$. Na medida em que se aumentou o volume de aplicação foi verificado aumento dos valores de deposição na entre linha das plantas. O mesmo foi observado nas linhas das plantas até a aplicação no volume de $160 \mathrm{~L} \mathrm{ha}^{-1}$. A presença do adjuvante na calda de pulverização não influenciou a deposição na linha e na entre linha da cultura do feijão.

PALAVRAS-CHAVE: Tecnologia de aplicação, contaminação por defensivos, retenção foliar.

\section{ABSTRACT}

Tank-mix adjuvants have the purpose to improve efficiency of pesticide applications. The aim of this research was evaluate different classes of agricultural adjuvants and application spray volume on bean plants deposits. The trials for determination of adjuvants effect on spray retention was carried out under greenhouse conditions and the trial aiming the interference of spray volume under field conditions. Different results regarding the deposition on bean plants were found depending to the type of adjuvant used. The adjuvant Haiten applied at dose of $100 \mathrm{~mL}^{100 \mathrm{~L}^{-1}}$

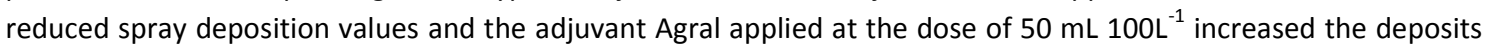
values. The increase in application volume provided higher deposition values on bean plant, but this increase was significant until the volume of $160 \mathrm{~L} \mathrm{ha}^{-1}$. As the spray volume increased, there was an increase on deposition values on collector placed between rows plants. The same was observed on the rows of the plants until the spray volume of $160 \mathrm{~L} \mathrm{ha}^{-1}$. The presence of the adjuvant in tank-mix did not influence deposition on the row and between the bean rows.

KEYWORDS: Application technology, pesticide contamination, foliar retention.

\section{RESUMEN}

Los adyuvantes son sustancias añadidas en la caldo de pulverización cuya finalidad es mejorar la eficiencia de las aplicaciones de defensivos agrícolas. La investigación tuvo como objetivos evaluar diferentes clases de adyuvantes agrícolas y del volumen de aplicación sobre los depósitos de la pulverización en plantas de frijol. El ensayo para determinar el efecto del adyuvante en la retención de la pulverización se realizó en invernadero y el ensayo para la interferencia del volumen de aplicación en condiciones de campo. Los resultados distintos en relación con la deposición de la pulverización en las plantas de frijol se encontraron a depender del tipo de adyuvante utilizado. El

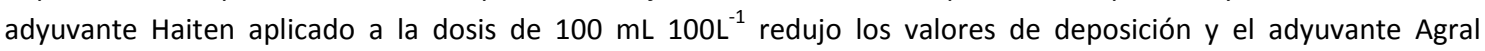
aplicado a la dosis de $50 \mathrm{~mL} 100 \mathrm{~L}^{-1}$ aumentó estos valores. El aumento del volumen de aplicación proporcionó mayores valores de deposición en las plantas de frijol, pero ese aumento fue significativo hasta el volumen de $160 \mathrm{~L}$ $\mathrm{ha}^{-1}$. La medida en que se aumentó el volumen de aplicación se verificó un aumento de los valores de deposición en la línea de las plantas. Lo mismo ocurrió en las líneas de las plantas hasta la aplicación del volumen de $160 \mathrm{~L} \mathrm{ha}{ }^{-1}$. La presencia del adyuvante en el caldo de pulverización no influenció la deposición en la línea y en la línea de cultivo.

PALABRAS-CLAVE: Tecnología de aplicación, contaminación por defensivos, retención foliar 


\section{INTRODUÇãO}

Uma das principais ferramentas para evitar as perdas ocasionadas por insetos e patógenos no feijoeiro, como em outras culturas, é a utilização de defensivos agrícolas os quais tem por função manter essas populações a níveis abaixo ao que causem danos econômicos. Embora, de acordo com o manejo integrado de pragas e doenças, o controle químico deve ser a última tática de controle é notória a necessidade dessa medida por não termos ainda hoje medidas de controle mais eficiente (SANTOS-JUNIOR et al., 2015).

Adjuvantes são acrescentados à calda de pulverização com o objetivo de melhorar a eficiência das aplicações de defensivos, reduzir o impacto das interferências ambientais e permitir uma penetração cuticular mais eficiente (QUEIROZ, 2008), facilitar o molhamento em superfícies hidrorepelentes e facilitar o contato da calda com a cutícula em superfícies pilosas que tendem a manter as gotas suspensas (KISSMANN, 1996).

O uso de adjuvantes redutores da tensão superficial da calda como os organosiliconados tem permitido aos produtores reduzir o volume de aplicação de defensivos agrícolas e aumentar a eficiência de controle de pragas e doenças em diversas culturas (GASKIN et al., 2002). A redução do volume de aplicação possui uma série de vantagens como: ingrediente ativo mais concentrado permitindo maior eficácia, maior rendimento dos pulverizadores, redução da quantidade de reabastecimento, redução da quantidade de água e possibilidade de aplicação de maior quantidade de área nas condições mais favoráveis a aplicação. Gotas provenientes de calda com baixa tensão superficial tem uma molhabilidade muito mais acentuada, cobrindo uma maior superfície onde essas foram aplicadas. Dessa forma a escolha de produtos tensoativos pode contribuir para que redução de volume de aplicação sem prejudicar a cobertura da pulverização.

A avaliação da pulverização pode ser feita por meio de um estudo da deposição de gotas sobre superfícies-alvo, que podem ser naturais ou artificiais. Existem vantagens e desvantagens quanto ao tipo de alvo a ser utilizado, no entanto, as superfícies naturais são mais utilizadas, por representarem melhor as condições reais de uma aplicação (MILLER, 1993). As avaliações dos depósitos de calda são utilizadas nas pesquisas de tecnologia de aplicação, como instrumento para desenvolver e melhorar as técnicas de aplicação de defensivos (PALLADINI, 2000).

A eficiência de defensivos agrícolas depende, dentre outros fatores, de uma cobertura adequada da calda pulverizada sobre as plantas sendo que essa cobertura pode ser influenciada pela quantidade de água utilizada durante o processo de aplicação (Knoche, 1994). Produtores preferem aplicar baixo volume com intuito de diminuir a quantidade de reabastecimento do pulverizador aumentando a capacidade operacional da máquina e dessa forma reduzindo os custos (SANTOS-JUNIOR et al., 2015). 


\section{REVISTA CIENTIFICA 2017}

\section{OBJETIVOS}

A pesquisa teve como objetivos avaliar diferentes classes de adjuvantes agrícolas e do volume de aplicação sobre os depósitos da pulverização em plantas de feijão.

\section{METODOLOGIA}

\section{Efeito de adjuvantes sobre os depósitos da pulverização}

O ensaio foi desenvolvido na Faculdade de Ciências Agrárias e Tecnológicas - UNESP, Câmpus de Botucatu. Cinco sementes de feijão foram semeadas na profundidade de $1 \mathrm{~cm}$ em vasos com 0,12 m de diâmetro e capacidade de 0,7 litros com duas partes de solo e uma parte de esterco bovino curtido. Após a emergência das plântulas foi realizado o desbaste permanecendo uma planta por vaso. As plantas permaneceram em estufa com ambiente não controlado sendo irrigadas diariamente até a capacidade de campo. As plantas foram submetidas aos tratamentos quando estavam no estádio vegetativo V3 ( 2 folhas trifolioladas). $O$ ensaio foi conduzido no delineamento inteiramente casualizados com 8 tratamentos e 10 repetições sendo cada repetição constituída por um vaso com uma planta. Os tratamentos

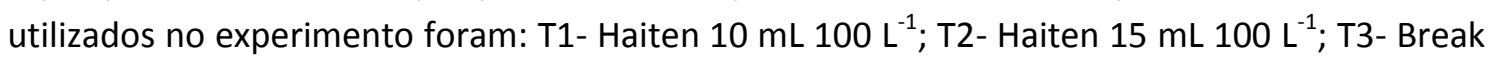
Thru 15 mL 100 L$^{-1}$; T4- Break Thru 100 mL 100 L$^{-1}$; T5- Agral 30 mL 100 L$^{-1}$; T6- Agral 50 mL 100 $\mathrm{L}^{-1}$; T7- Assistl $500 \mathrm{~mL} 100 \mathrm{~L}^{-1}$; T8- Testemunha (sem adjuvante). O corante Azul Brilhante na concentração de $1,5 \mathrm{~g} \mathrm{~L}^{-1}$ diluídos em água deionizada foram utilizados em todos os tratamentos.

As pulverizações dos tratamentos foram realizadas através de pulverizador pesquisa (Herbicat ${ }^{\circledR}$ ) pressurizadas por $\mathrm{CO}_{2}$ equipados com 4 pontas espaçadas em $0,5 \mathrm{~m}$ aplicando em velocidade constante de $5 \mathrm{~km} \mathrm{~h}^{-1}$. As pontas utilizadas no trabalho foram de jato plano modelo XR 8002 (Teejet ${ }^{\oplus}$ ) regulado para proporcionar um volume de aplicação de aproximadamente $160 \mathrm{~L} \mathrm{ha}{ }^{-1}$. Todos os tratamentos receberam o mesmo volume de aplicação. As condições ambientais como temperatura e umidade relativa do ar foram monitoradas no momento das pulverizações através de Termo-higro-anemômetro digital portátil (modelo THAL-300). A pulverização foi realizada dentro de uma casa de vegetação evitando dessa forma a interferência pelo vento. As características dos adjuvantes empregados no estudo estão apresentadas na Tabela 1 


\section{ANAP Brasil \\ ISSN 1984-3240 \\ v. 10, n. 21}

\section{REVISTA CIENTIFICA 2017}

Tabela 1. Caracterização dos adjuvantes estudados

\begin{tabular}{|c|c|c|c|c|}
\hline $\begin{array}{c}\text { Marca } \\
\text { comercial }\end{array}$ & $\begin{array}{l}\text { Ingrediente ativo } \\
\text { (i.a.) }\end{array}$ & $\begin{array}{c}\text { Concentração } \\
\text { i.a. }\end{array}$ & $\begin{array}{c}\text { Dose } \\
\text { recomendada }\end{array}$ & Formulação \\
\hline Haiten & polioxietileno aquil fenol éter & $800 \mathrm{~g} \mathrm{~L}^{-1}$ & $10-15{\mathrm{~mL} 100 \mathrm{~L}^{-1}}^{-1}$ & $\begin{array}{c}\text { Concentrado } \\
\text { solúvel }\end{array}$ \\
\hline Break Thru & $\begin{array}{l}\text { copolímero de poliéter e } \\
\text { silicone }\end{array}$ & $1000 \mathrm{~g} \mathrm{~L}^{-1}$ & $15-100 \mathrm{~mL} 100 \mathrm{~L}^{-1}$ & $\begin{array}{c}\text { Concentrado } \\
\text { dispersível }\end{array}$ \\
\hline Agral & noni poli (etilenoxi) etanol & $200 \mathrm{~g} \mathrm{~L}^{-1}$ & 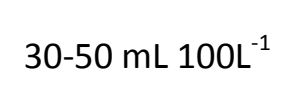 & $\begin{array}{c}\text { Concentrado } \\
\text { solúvel }\end{array}$ \\
\hline Assist & óleo mineral & $756 \mathrm{~g} \mathrm{~L}^{-1}$ & 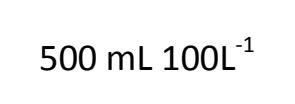 & $\begin{array}{l}\text { Concentrado } \\
\text { emulsionável }\end{array}$ \\
\hline
\end{tabular}

Fonte: Agrofit (2017)

\section{Quantificação dos depósitos da pulverização}

Após a aplicação dos tratamentos as plantas foram cortadas rente ao solo por tesouras e acondicionadas em sacos plásticos (1L) e em seguida lavadas com $100 \mathrm{~mL}$ de água deionizada para remoção do corante. A solução contendo o corante foi alocada em potes plásticos de 70 $\mathrm{mL}$ de capacidade. Depois de lavadas, as plantas foram secadas com auxílio de papel toalha, colocadas em sacos de papel etiquetados e levadas a uma estufa de circulação e renovação de ar (TE - 394/3), para secagem. Após 72 horas, a uma temperatura de $65{ }^{\circ} \mathrm{C}$, as plantas foram retiradas e pesadas, determinando-se a massa seca (MS) em balança analítica. A quantidade do corante depositada nas plantas foi quantificada em espectrofotômetro (Bioespectro modelo SP 220) com comprimento de onde $630 \mathrm{~nm}$ (SCUDELER et al., 2004). Com as concentrações prévias já conhecidas do corante de 10,0; 5,0; 2,5; 1,25; 0,625 e 0,15625 mg L ${ }^{-1}$ foi determinado a equação de reta linear que permitiu transformar os valores de absorbância em concentração do corante $\left(\mathrm{mg} \mathrm{L}^{-1}\right)$. Em virtude de algum possível efeito do adjuvante na coloração da solução foi determinado à equação da reta para cada solução contendo adjuvante. De posse dos valores de concentração do corante na calda, concentração do corante na planta e volume de diluição da amostra foi possível estabelecer o volume capturado pelo alvo através da equação:

$\mathrm{V}_{1}=\left[\mathrm{C}_{2} \cdot \mathrm{V}_{2}\right] / \mathrm{C}_{1}$

Em que:

$\mathrm{V}_{1}=$ volume retido pelo alvo $(\mathrm{mL}) ;$

$\mathrm{C}_{2}=$ concentração detectada no espectrofotômetro $\left(\mathrm{mg} \mathrm{L}^{-1}\right)$;

$\mathrm{V}_{2}=$ volume de diluição da amostra de cada planta $(\mathrm{mL})$;

$\mathrm{C}_{1}=$ concentração inicial na calda de aplicação $\left(1500 \mathrm{mg} \mathrm{L}^{-1}\right)$.

$O$ volume retido em cada planta em $\mathrm{mL}$ foi dividido pela sua respectiva $\mathrm{MS}$, obtendose assim a quantidade em $\mathrm{mL} \mathrm{g}^{-1}$ de MS. 


\section{ANAP Brasil \\ ISSN 1984-3240 \\ v. 10, n. 21}

\section{REVISTA CIENTIFICA 2017}

\section{Determinação dos depósitos da pulverização e perdas para o solo em função do volume de aplicação}

O experimento foi conduzido na área experimental pertencente à Agência Paulista de Tecnologia do Agronegócio (Apta) polo regional extremo Oeste. A semeadura do feijão foi realizada, no espaçamento de $0,45 \mathrm{~m}$ entre linhas e densidade populacional de aproximadamente 10 plantas $\mathrm{m}^{-1}$ linear em sistema de semeadura direta no solo. A cultivar utilizada foi a IAC Milênio semeada na primeira quinzena de julho. Irrigações por aspersão foram realizadas conforme a necessidade da cultura. $O$ ensaio foi conduzido no delineamento experimental inteiramente casualizado com 8 tratamentos e 4 repetições no esquema fatorial $4 \times 2$ (volume de aplicação de 60,110, 160 e $210 \mathrm{~L} \mathrm{ha}^{-1}$ ) com e sem adjuvante Haiten na

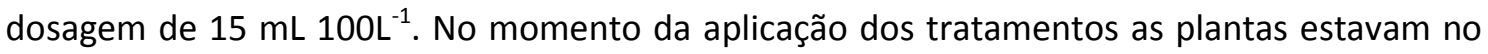
estádio de desenvolvimento reprodutivo R8 (enchimento de vagens). Cada parcela constou de $3 \mathrm{~m}$ de largura por $7 \mathrm{~m}$ de comprimento. Para quantificar as perdas por escorrimento foram colocados na linha e entre linha das plantas 20 potes plásticos (capacidade de $70 \mathrm{~mL}$ e diâmetro de $11 \mathrm{~cm}$ ) por tratamento (10 na linha e 10 na entrelinha).

Figura 1. Detalhe dos coletores posicionados na entre linha (a) e na linha (b) da cultura do feijão no momento da aplicação dos tratamentos.
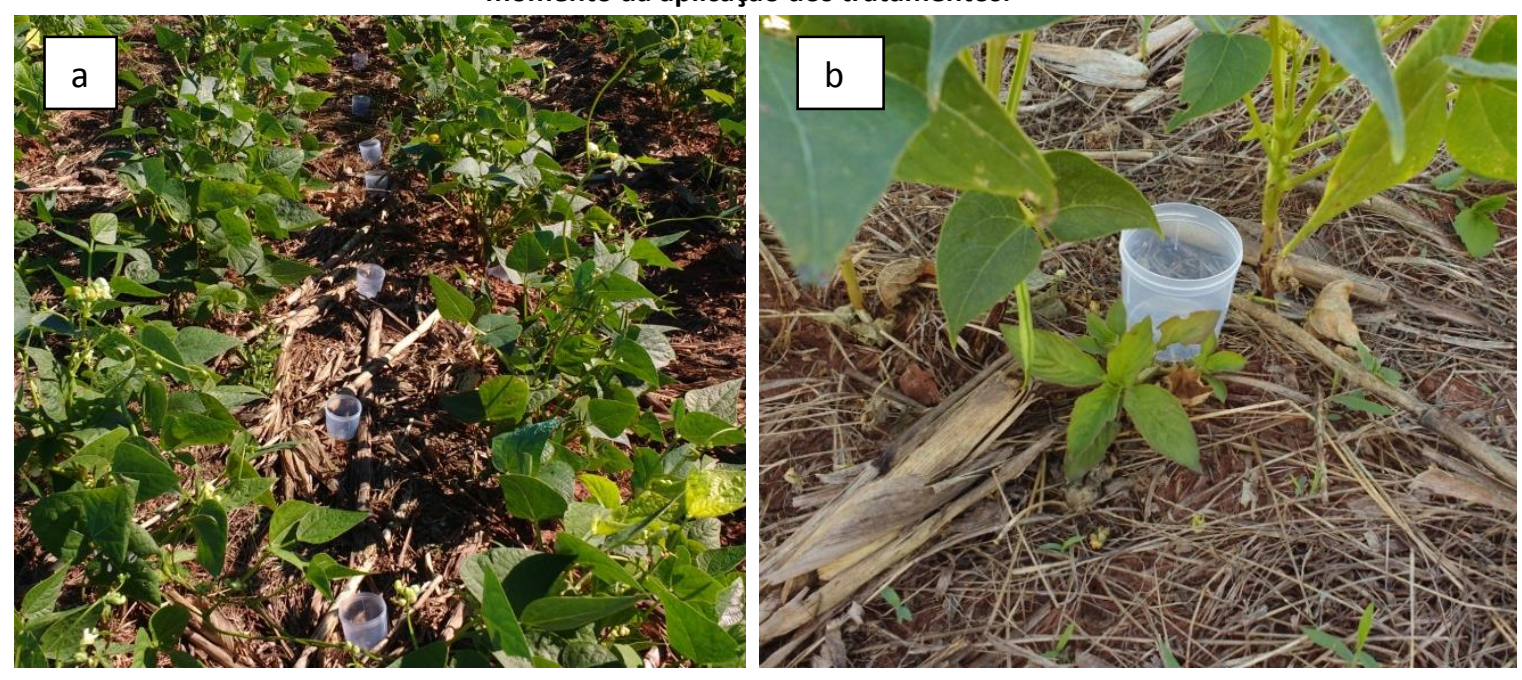

As pulverizações foram realizadas através de pulverizador pesquisa (Herbicat ${ }^{\circledR}$ ) pressurizadas por $\mathrm{CO}_{2}$ equipados com 6 pontas espaçadas em $0,5 \mathrm{~m}$. As pontas utilizadas no trabalho foram de jato plano modelo XR 8001, 8002 e 8003 (Teejet ${ }^{\circledR}$ ) e pressões variando de 1 a 3 bar a depender do volume de aplicação desejado. No momento da aplicação foram monitoradas as condições ambientais como descrita no item 3.1.

Após secagem da calda pulverizada coletou-se 7 plantas por parcela e essas separadas em partes superior e inferior. As partes das plantas foram acondicionadas em sacos plásticos (3L) previamente identificados e levados ao laboratório. No laboratório foram realizados os procedimentos descritos no item 3.3. 


\section{ANAP Brasil

\section{REVISTA CIENTIFICA 2017}

\section{Análise estatística dos dados}

Os valores dos depósitos da pulverização, para ambos os ensaios, foram submetidos à análise de variância sendo as médias comparadas pelo teste LSD a $5 \%$ ou a $10 \%$ de probabilidade.

\section{RESULTADOS E DISCUSSÕES}

\section{Efeito de adjuvantes sobre os depósitos da pulverização}

Os dados médios de deposição da calda de pulverização em plantas de feijão expressos em $\mu \mathrm{L}$ $\mathrm{g}^{-1} \mathrm{MS}$ são descritos na Figura 2. Resultados com menores valores de deposição foram

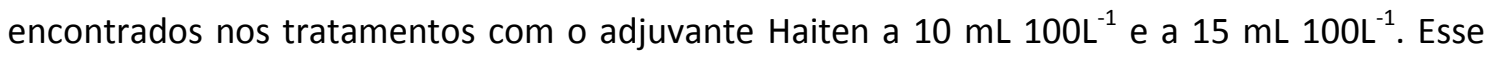
adjuvante nas duas doses testadas apresentou media de deposição significativamente menores comparadas com os adjuvantes Break Thru e Agral em ambas as doses testadas (Figura 2).

O tratamento que apresentou maior valor de deposição foi o Agral a $50 \mathrm{~mL}^{100 \mathrm{~L}^{-1}}$, sendo o único a diferir significativa em relação ao tratamento onde foi não teve a adição de adjuvante (testemunha). Para os demais tratamentos, não houve diferença significativa em relação à testemunha.

Figura 2. Valores de deposição em plantas de feijão em função da pulverização de diferentes adjuvantes.

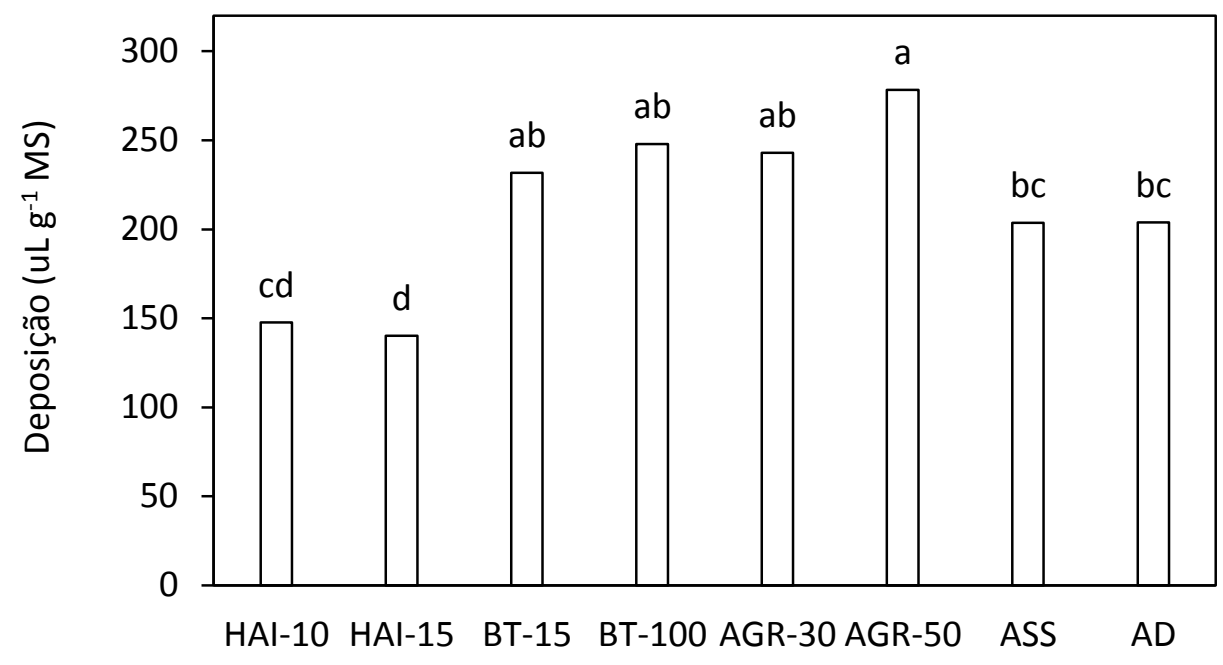

Adjuvantes

Médias seguidas pela mesma letra não difere signficativamente entre si pelo teste LSD $(p<0.1)$.

HAI-10: Haiten $10 \mathrm{~mL} 100 \mathrm{~L}^{-1}$; HAl-15: Haiten $15 \mathrm{~mL} 100 \mathrm{~L}^{-1}$; BT-15: Break Thru $15 \mathrm{~mL} 100 \mathrm{~L}^{-1}$; BT-100: Break Thru 100 $\mathrm{mL} 100 \mathrm{~L}^{-1}$; AGR-30: Agral $30 \mathrm{~mL} 100 \mathrm{~L}^{-1}$; AGR-50: Agral $50 \mathrm{~mL} 100 \mathrm{~L}^{-1}$; ASS: Assist $50 \mathrm{~mL} 100 \mathrm{~L}^{-1}$; AD: Água deionizada. Foi adicionado o corante azul brilhante na concentração de $1,5 \mathrm{~g} \mathrm{~L}^{-1} \mathrm{em}$ todos os tratamentos. 
A utilização de adjuvantes na calda de pulverização é prática rotineira por muitos agricultores. Essas substâncias têm como intuito aumentar a eficiência do defensivo agrícola ou modificar certas propriedades da calda facilitando o processo de aplicação. Umas das características dos adjuvantes redutores da tensão superficial (espalhante adesionante) é proporcionar o aumento da quantidade depositada da calda na superfície dos vegetais (HOLLOWAY et al., 2010). Como verificado na Figura 2, comportamento divergentes quanto a essa propriedade são verificados, sendo que existem alguns adjuvantes que proporcionam aumento da quantidade de depósitos enquanto que outros proporcionem efeitos contrários. Vale lembrar que o tipo de superfície vegetal desempenha importante papel nessas propriedades e que distintos resultados podem variar não somente pela alteração das características do adjuvante, mas também com o tipo de planta a ser estudado.

$\mathrm{O}$ aumento da deposição dos defensivos sobre as plantas pode proporcionar maiores eficiência de controle e, dessa forma, possibilitando a redução da quantidade de ingrediente ativo (i.a.) do defensivo. A redução da quantidade de defensivo aplicado gera uma redução dos custos de produção além da minimização dos possíveis problemas que esses agentes podem ocasionar no ambiente.

\section{Determinação dos depósitos da pulverização e perdas para o solo em função do volume de aplicação}

A análise de variância detectou diferença significativa $(F=101,8 ; p<0,001)$ para volume de aplicação, $(F=5,3 ; p=0,02)$ para adjuvante. Não foi verificada diferença significativa para a interação volume de aplicação $x$ adjuvante $(F=1,3 ; p=0,27)$.

Na Figura 3 são apresentados os resultados de deposição em função do volume de aplicação. Os volumes de aplicação de 160 e $210 \mathrm{~L} \mathrm{ha}^{-1}$, foram os que obtiveram os maiores valores de deposição diferenciando significativamente dos volumes de 60 e $110 \mathrm{~L} \mathrm{ha}^{-1}$, porém não diferenciando entre si. $O$ volume de aplicação de $210 \mathrm{~L} \mathrm{ha}^{-1}$ não promoveu incremento de deposição provavelmente pela planta ter atingido a capacidade máxima de retenção e consequentemente ocasionado o escorrimento do excesso da calda. Resultados semelhantes encontrados nesse trabalho foram reportados por Santos Júnior et al. (2015) onde esses autores relataram aumento nos depósitos da pulverização pelo aumento do volume de aplicação até certo volume e que a partir de então o aumento do volume de aplicação não resultava em expressivo aumento de deposição.

$\mathrm{O}$ volume de $60 \mathrm{~L} \mathrm{ha}^{-1}$ foi o que apresentou as menores médias de deposição diferenciando significativamente dos demais volumes de aplicação (Figura 3). Fica evidente que o aumento do volume aplicação proporcionou incrementos da quantidade de depósitos nas plantas de feijão até o volume de $160 \mathrm{~L} \mathrm{ha} a^{-1}$. Volumes de aplicação superiores a $160 \mathrm{~L} \mathrm{ha}^{-1}$ não seriam recomendados, pois ultrapassaria a capacidade de retenção das plantas de feijão, como mencionado anteriormente, e dessa forma, desperdício de defensivos, redução da dose efetiva do ingrediente ativo sobre a planta e consequentemente contaminação do ambiente. 
Uma tendência de redução do volume de aplicação, por parte dos agricultores, vem crescendo nos últimos anos em virtude da redução dos custos operacionais da aplicação pelo aumento da capacidade operacional dos pulverizadores. Como exemplo, um tanque de um pulverizador com capacidade de $600 \mathrm{~L}$ se regulado para proporcionar uma vazão de $60 \mathrm{~L} \mathrm{ha}^{-1}$ tem capacidade de tratar uma área de 10 hectares. Para esse mesmo pulverizador, regulado para aplicar na vazão de $210 \mathrm{~L} \mathrm{ha}^{-1}$ só conseguiria aplicar uma área de aproximadamente 2,9 hectares.

Cuidados com a redução no volume de aplicação devem ser tomados para que não possa reduzir a eficiência de controle do defensivo. Nessa pesquisa, verifica-se que os depósitos da pulverização quando aplicado com volume $60 \mathrm{~L} \mathrm{ha}^{-1}$ teve uma redução de aproximadamente 3 vezes quando comparado com os depósitos da aplicação dos maiores volumes (160 e $210 \mathrm{~L}$ ha' ${ }^{1}$ ). Vale lembrar que o volume de aplicação é somente o veículo que carrega o i.a. dos produtos e que nem sempre, menores valores de deposição estão relacionados com os menores valores de i.a. depositado na planta. Pesquisas relacionadas com a eficiência de controle de defensivos aplicados em volumes reduzidos devem ser conduzidas para um posicionamento mais assertivo dessa prática.

Figura 3. Valores médios de deposição em plantas de feijão em função da pulverização com diferentes volumes de aplicação.

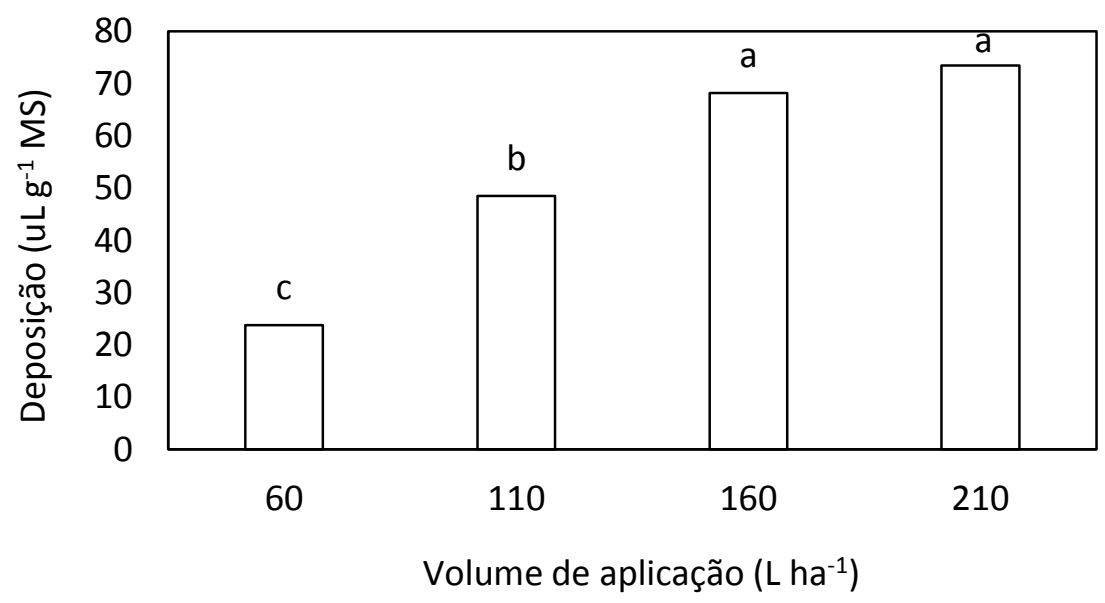

Médias seguidas pela mesma letra não difere signficativamente entre si pelo teste LSD a $5 \%$ de probabilidade.

A Figura 4 apresenta os valores médios de deposição em função da aplicação realizada com e sem o adjuvante Haiten. Verifica-se que maior valor de deposição da calda quando a pulverização foi realizada sem adjuvante. Embora visualmente essa diferença não seja tão expressiva, foi suficiente para proporcionar diferença significativa. Os resultados obtidos em campo corroboram com os resultados obtidos em estuda em que o adjuvante Haiten aplicado na dose de $15 \mathrm{~mL} 100 \mathrm{~L}^{-1}$ proporcionou menores resultados de deposição comparados com a aplicação somente com água deionizada. 


\section{ANAP Brasil \\ ISSN 1984-3240 \\ v. 10, n. 21}

\section{REVISTA CIENTIFICA 2017}

Figura 4. Valores médios de deposição dos tratamentos com e sem adjuvante Haiten.

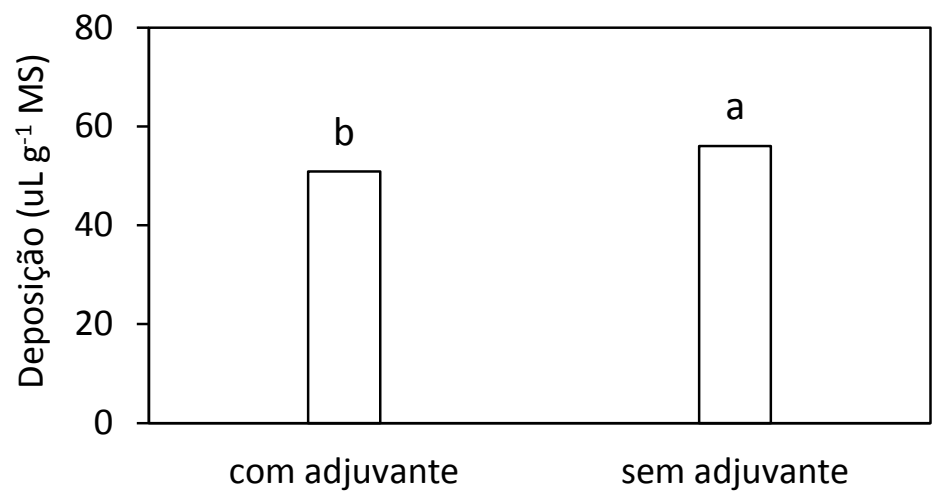

Médias seguidas pela mesma letra não difere signficativamente entre si pelo teste LSD a 5\% de probabilidade.

A Figura 5 apresenta os valores médios de deposição na entre linha e na linha de feijão em função do volume de aplicação. Verifica-se maiores valores de deposição nos coletores posicionados na entre linha das plantas comparados com os coletores posicionados na linha. Quando foi realizada a aplicação do corante o dossel da cultura do feijão não estava fechado permitindo uma maior deposição na região da entre linha. Já para os coletores posicionados na linha da cultura, menores valores de deposição foram encontrados devido o efeito guardachuva proporcionado, principalmente, pelas folhas de feijão (Figura 1).

Maiores valores de deposição na entre linha das plantas foram encontrados quando a aplicação foi realizada com os maiores volumes de aplicação. A medida que aumentou o volume de aplicação foi verificado maiores valores de deposição. Resultados semelhantes de deposição foram encontrados nos coletores posicionados na linha das plantas onde o aumento do volume de aplicação proporcionou os maiores valores de deposição. Esse aumento só não foi verificado na aplicação com o volume de $210 \mathrm{~L} \mathrm{ha}^{-1}$ que não diferenciou significativamente do volume de $160 \mathrm{~L} \mathrm{ha}^{-1}$. 


\section{REVISTA CIENTIFICA 2017}

Figura 5. Valores médios de deposição na linha e entrelinha das plantas de feijão em função do volume de aplicação.

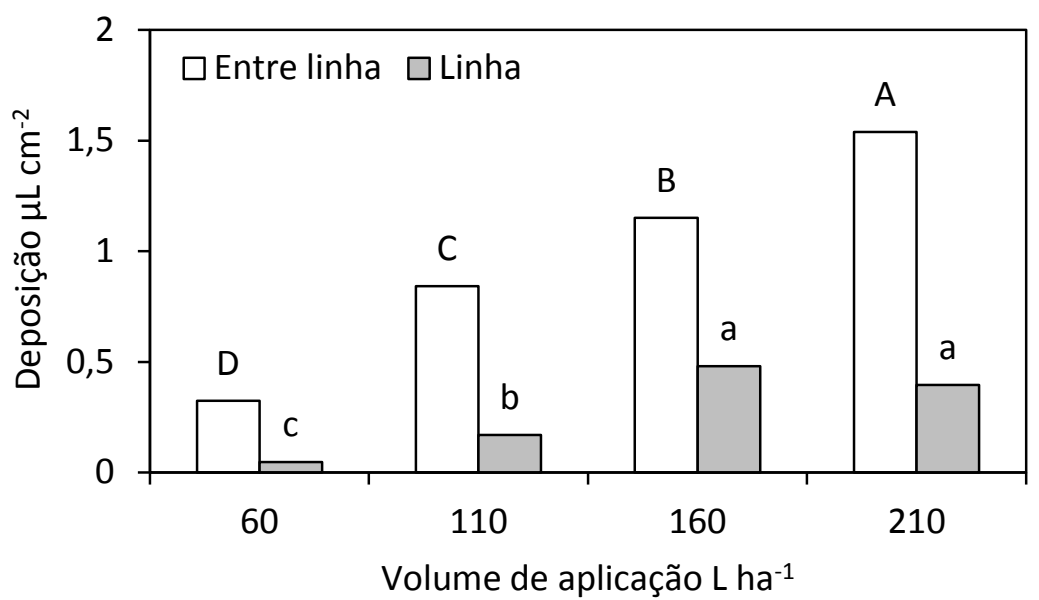

Letras maiúsculas e minúsculas comparam deposição na entrelinha e linha respectivamente. Médias com mesma letra não difere significativamente entre si pelo teste LSD a $5 \%$ de probabilidade.

A Figura 6 apresenta os valores médios de deposição na linha e entrelinha das plantas de feijão com sem a adição de adjuvante Haiten na calda de aplicação. Não foi verificado diferença significativa na linha e entre linha de plantas de feijão nas aplicações realizas com e sem adjuvante.

Figura 6. Valores médios de deposição na linha e entrelinha das plantas de feijão com sem a adição de adjuvante Haiten na calda de aplicação.

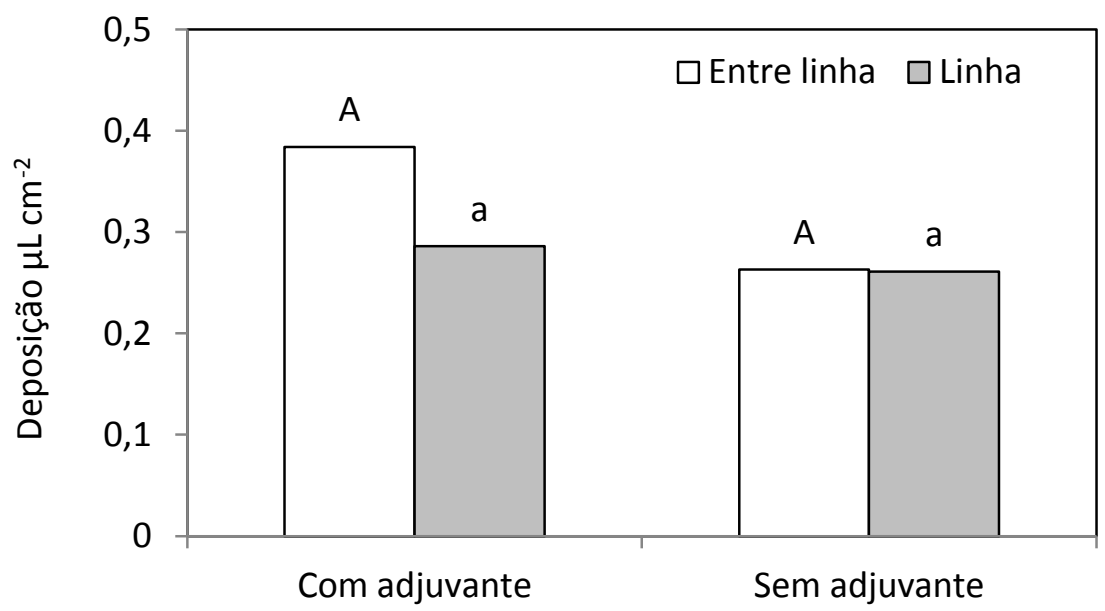

Letras maiúsculas e minúsculas comparam deposição na entrelinha e linha respectivamente. Médias com mesma letra não difere significativamente entre si pelo teste LSD a $5 \%$ de probabilidade. 


\section{ANAP Brasil \\ ISSN 1984-3240 \\ v. 10, n. 21}

\section{REVISTA CIENTIFICA 2017}

\section{CONCLUSÃO}

Os adjuvantes utilizados nessa pesquisa apresentaram resultados distintos em relação a deposição da calda em plantas de feijão. $O$ adjuvante Haiten aplicado na dose de $100{\mathrm{~mL} 100 \mathrm{~L}^{-}}^{-}$ ${ }^{1}$ reduziu os valores de deposição e o adjuvante Agral aplicado na dose de $50 \mathrm{~mL} 100 \mathrm{~L}^{-1}$

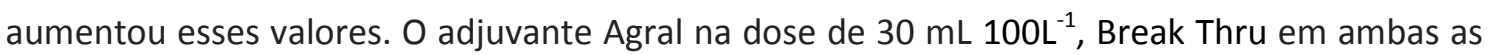
doses e Assist não apresentaram diferença de deposição quando comparado com a aplicação com água deionizada. Essa diferença de deposição proporcionada pela adição dos adjuvantes provavelmente está relacionada com as propriedades intrínsecas de cada produto. $\mathrm{O}$ aumento do volume de aplicação proporcionou maiores valores de deposição nas plantas de feijão, porém esse aumento foi significativo até o volume de $160 \mathrm{~L} \mathrm{ha}^{-1}$. À medida que se aumentou o volume de aplicação foi verificado aumento dos valores de deposição na entre linha das plantas. O mesmo aconteceu nas linhas das plantas até a aplicação do volume de $160 \mathrm{~L} \mathrm{ha}^{-1}$. A presença do adjuvante na calda de pulverização não influenciou a deposição na linha e na entre linha da cultura.

\section{REFERÊNCIAS BIBLIOGRÁFICAS}

GASKIN, R.E.; MANKTELOW, D.M.L.; SKINNER, S.J.; G.S. ELLIOTT. use of a superspreader adjuvant to reduce spray application volumes on avocados. New Zealand Plant Protection, v.57, p.266-270, 2004.

HOLLOWAY, P.J.; ELLIS, M.B.; WEBB, D.A.; WESTERN, N.M.; TUCK, C.R.; HAYES, A.L.; MILLER, P. C. H. Effects of some agricultural tank-mix adjuvants on the deposition efficiency of aqueous sprays on foliage. Crop Protection, v.19, n.1, p.27-37, 2000.

KISSMANN, K.G. Adjuvantes para caldas de defensivos agrícolas. BASF: São Paulo, 1996. 45p.

MILLER, P. C. H. Spray drift and its measurement. In: MATTHEWS, G. A.; HISLOP, E. C. Application technology for crop protection. Trowbridge: CAB International, 1993. p. 101-122.

PALLADINI, L. A. Metodologia para avaliação da deposição em pulverizações. 2000. $111 \mathrm{f}$. Tese (Doutorado em Agronomia/Proteção de Plantas) - Universidade Estadual Paulista, Botucatu, 2000.

QUEIROZ, A. A.; MARTINS J.A.S.; CUNHA, J. P. R.; Adjuvantes e qualidade da água na aplicação de agrotóxicos. Bioscience Journal (UFU), v. 24, p. 8-19, 2008.

SANTOS-JÚNIOR, J. L. C.; SANTOS, N. C. B.; CAÇÃO, J. E. C. R.; DONDE, S. C.; PRADO, E.P. Desempenho de diferentes volumes de aplicação na deposição em feijão. In: Simpósio de Iniciação Científica da Unesp de Dracena - SICUD, 2015.

SCUDELER, F. et al. Cobertura da pulverização e maturação de frutos do cafeeiro com ethephon em diferentes condições operacionais. Bragantia, Campinas, v.63, n.1, p.129-139, 2004 